\title{
Oscillation of gear mechanisms of construction machines
}

\author{
Vladimir Skel $^{*}$ \\ Moscow State University of Civil Engineering, Yaroslavskoe shosse, 26, Moscow, 129337, Russia
}

\begin{abstract}
The article is devoted to the study of the influence of gearing errors on the vibration activity of the open gear transmission of construction machines. A brief review of the literature on the relevance of the topic of gear dynamics in different industries and the results achieved so far is given. The features of open gears of turning mechanisms of construction machines are highlighted: a large gear ratio, a large gearing module, the absence of a common frame for the gear and wheel, poor transmission lubrication conditions, a small degree of precision in the manufacture of gears and the actual overlap coefficient is close to one. With these features, the main internal source of vibration is the difference between the main steps of the gear and the wheel and the error of the involute profile of the teeth. The results of the study of the influence of these features of vibration damping and open gear transmission on the dynamics of the turning mechanism of the construction machine are presented. The studies were carried out on a generalized two-mass nonlinear dynamic model with internal perturbation from the gear pair and with structural damping. The studies were conducted mainly for the transitional modes of resonance. Based on the analysis of the results obtained and presented in the article, recommendations are given to reduce the fluctuations of the turning mechanisms of construction machines.
\end{abstract}

\section{Introduction}

A large amount of work in the construction of civil and industrial buildings, as well as in the construction of hydraulic engineering and other structures, is carried out by construction machines. The problems of the dynamics of construction machines are considered in many works, for example, [1]. The performance of construction machines depends on the performance of all their elements [2]. Many construction machines have a mechanism of rotation of the movable part (platform for excavator or construction crane, tower or flat-top tower crane and other machines), relatively fixed parts (undercarriage parts for excavator or construction crane undercarriage or fixed tower tower crane and so on) [3]. This mechanism most often consists of a simple or planetary gearbox and an open gear drive. The open gear consists of a gear with a small number of teeth connected together with the gearbox to the rotary part of the machine, and a gear ring with a large number of teeth connected to the stationary part of the machine. The open transmission of turning

\footnotetext{
*Corresponding author: SkelVI@mgsu.ru
} 
mechanisms of construction machines, in comparison with other mechanisms, has design features: it does not have a casing that isolates the transmission from the external environment; a large gear ratio compared to a conventional single-stage transmission; a relatively large module of involute engagement; poor transmission lubrication conditions; a small degree of precision of gears and gears. To these features, you can add that the wheel has a large size (more than one meter in diameter) and the actual overlap coefficient in the real gearing is close to one. The experience of operation and research of construction machines has shown that the open gear transmission relatively often fails and one of the reasons for this is the dynamics of the transmission [4].

In [5], I considered the relative values of perturbations that occur in an open gear train. Were considered: the difference between the basic steps of the gear teeth and the wheels; the error margin involute tooth profile; misalignment and skewed relative position of the axes of the gears and wheels that occur due to the lack of a common frame will have wheels; force disturbance from the forces of friction in the engagement; variable stiffness of engagement with one-pair and two-pair engagement.

\section{Literature review}

Many papers have been devoted to the problem of gear dynamics, starting with [6, 7]. Despite such a long history of research, the problem under consideration exists in the mechanisms and machines of many industries. This is due to the new level of technology development and new requirements for the performance of mechanisms and machines. For example, there are new construction materials, more intensive modes of operation with higher speeds. In many modern works, the problem of the dynamics of gears is posed as a nonlinear one $[8,9,10]$. In this regard, in these works and others, much attention is paid to methods for solving problems of dynamics: electronic modeling and finite element methods. In this case, particular problems of the causes of vibration activity of gears can be solved. For example in [10] considers modeling the disturbance of sliding friction in contact; in $[11,12,13]$ considered the variable rigidity of the gear in the presence of multiple cracks in the stem of the tooth, with the issues of modeling and use the results to diagnose gear; In [14], a model is also considered taking into account the influence of stiffness, tooth modification, and damping due to the presence of lubrication between the teeth; in [15], the nonlinear dynamics of variable gearing stiffness and a method for solving such a problem are considered; the article [16] is devoted to the solution of a nonlinear model of gear transmission operation with backlash and internal and external perturbation. There are works devoted to reducing the dynamic loads in gears, for example, by changing the malleability of the teeth [17] or modifying the engagement and rigidity of the gearbox housing [18]. The article [19] notes the importance of determining the dynamics of gears at the stage of their design and provides some critical review of methods for modeling dynamic systems.

These and other works and the analysis of failures of gears of machines and mechanisms showed the relevance of the issues of the dynamics of gears.

\section{Contents of the research}

From literature and our own studies [5] show that vibroactivity gear arise for several reasons: the difference between basic steps gears and wheels; the distortion of the involute tooth profile; variable stiffness gearing; friction in the gearing and some other reasons. Of all the listed causes of vibrations, the greatest disturbance comes from the difference in the main steps of the gear and the wheel and the distortion of the involute profile of the teeth. In [5], we also present a generalized dynamic model (Figure 1) of oscillations of the rotation mechanism of a single-bucket excavator and the nature of nonlinear equations that 
reflect the oscillations of this mechanism under the action of internal perturbation from the gear train. All the characteristics of the model are selected for the parameters of the rotation mechanism of the single-bucket excavator. Affiliations of authors should be typed in 9point Times. They should be preceded by a numerical superscript corresponding to the same superscript after the name of the author concerned. Please ensure that affiliations are as full and complete as possible and include the country.

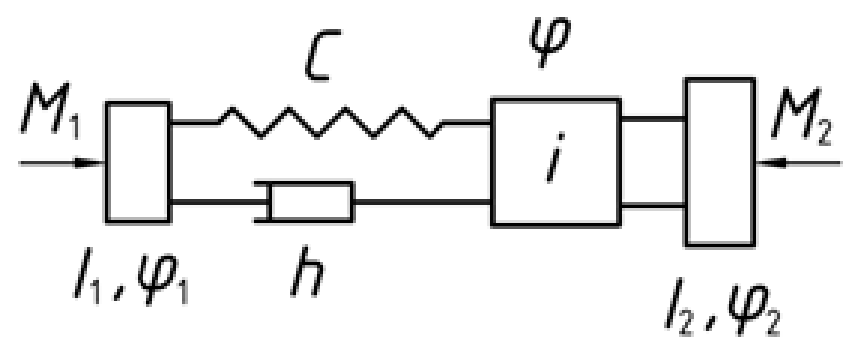

Fig. 1. Generalized dynamic model of the excavator turning mechanism.

In Fig. 1 marked: $\mathrm{J} 1$ is the moment of inertia of the engine; $\mathrm{J} 2$ is the moment of inertia of the turntable; With the rigidity of the rotation mechanism shovel; $h$ - generalized viscosity coefficient in the mechanism (mainly structural damping); M1, M2 -the momentum and the moment of resistance; - the coordinates of the mass movement J1, J2; i-gear ratio ring pair; - additional coordinate-movement of the massless gear.

The nature of the equations in dimensionless coordinates was as follows:

$$
\left\{\begin{array}{l}
\ddot{x}_{1}+b \dot{x}_{1}+x_{1}=-\mu \cdot \phi\left(x_{1}, x_{2}, \dot{x}_{1}, \dot{x}_{2}, \gamma, b\right)+a_{1} \\
\ddot{x}_{2}=\mu a_{2} \cdot \phi\left(x_{1}, x_{2}, \dot{x}_{1}, \dot{x}_{2}, \gamma, b\right)+a_{3}
\end{array},\right.
$$

where $\phi\left(x_{1}, x_{2}, \dot{x}_{1}, \dot{x}_{2}, \gamma, b\right)$ is the function that depends on the coordinates $\mathrm{x} 1$ and $\mathrm{x} 2$, their velocities, damping properties of the system $\mathrm{b}$ and the phase of the perturbation $\gamma$; $\mu$ is the relative amplitude of the kinematic perturbation; a1, a2, a3 are the constant components that depend on the inertial, stiffness and force (moments of driving and resistance) parameters of the system.

As a continuation of the work [5], a study was carried out in relation to the excavator, in which the influence of the excavator parameters on the amplitude of the relative mass fluctuations of the dynamic system is considered. Since the dynamical system is nonlinear, its stability was investigated. For this purpose, the equations of free oscillations of the platform motion are considered and analyzed. The most important mode of possible transient resonance for the resulting loads was also considered: the resonant zone is passed at the speed of rotation of the turntable. This mode corresponds to the actual operating mode of the excavator.

Nonlinear differential equations were integrated by the numerical Runge-Kutta method. The coordinate of the relative mass motion of the system is chosen as the main one, and the results are presented for this coordinate. In this study, a graphical solution method was used in the stroboscopic phase plane [20].

\section{Results and comment}

1. The study of the stability of the selected nonlinear dynamic model with internal perturbation for the parameters of the turning mechanism of the excavator's turntable showed that the system has a tendency to self-oscillation. Taking into account the parameters of single-bucket excavators, the system has asymptotic stability. 2 . The study of free oscillations. The influence of the system parameters on the amplitude of the largest 
oscillations was determined, first of all, to change the value of the gear ratio in the form of a coefficient $\mu$. In Figure 2 for example, the nature of the graphs in the stroboscopic plane is shown for the case of free oscillations under the initial conditions of movement of the turntable.

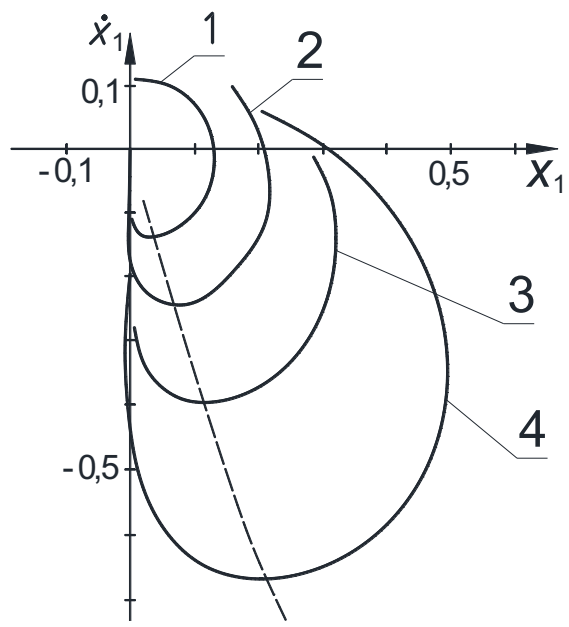

Fig. 2. Influence of the amplitude of the change in the gear ratio.

$1-\mu=0,005 ; 2-\mu=0,01 ; 3-\mu=0,02 ; 4-\mu=0,05$.

Figure 2 shows that the dependence of the amplitude of the largest oscillations $\mathrm{x} 1$ on the amplitude of the change in the gear ratio is nonlinear. In this case, the nonlinear system of equations tends to be linear, which in the stroboscopic plane is represented by a point (in this case, the origin).

Effect of structural damping "b "(depending only on the deformation rate of the mechanism ) The effect on the amplitude of the oscillations is also nonlinear. At $b=0$, continuous almost periodic oscillations of the beat type occur. With an increase in the coefficient "b", the amplitude of the oscillations decreases, and the pattern of oscillations becomes the same as when a linear system passes through a resonance at a low speed.

The study of the influence of the mass ratio during structural damping showed that the minimum amplitudes of the mechanism vibrations correspond to the case when the given "masses" J1 and J2 in Figure 1 are equal to each other. Deviation from this equality in any direction leads to an increase in the amplitude of the oscillations.

The influence of other parameters was also investigated, for example, the phase of action of the error of the gear ratio: it turned out that it does not affect the amplitude of free oscillations.

3. The study of non-free oscillations. The perturbation from the variable transfer ratio was represented as a binomial expansion of the perturbation function into a Fourier series. The study of the system of differential equations of the generalized dynamic model showed that it has no stationary motion ( $a t=0$, the system has orbital stability [21]). Therefore, we may be interested in the stability of possible periodic (almost periodic) oscillations. The phase diagrams given in paragraph 3.3.2 show: 1) for the parameters of a generalized dynamic system typical of single-bucket excavators, the oscillations with maximum amplitudes are unstable, although the amplitude change is very slow; 2) at $\mathrm{J} 1 \rightarrow 0$, the system behaves as if it were forced to oscillate; 3 ) it is possible to choose the magnitude and shape of the change in the driving moment so that the oscillations of the system with the maximum little fluctuating amplitude will be stable; 4) vibration damping due to the electric drive may be more significant than structural damping (the greater the rigidity of the mechanical characteristics of the drive, the greater the damping effect); 5) the amplitude of vibrations at 
transient resonance depends on the rate of passage of the resonant zone; if we take into account the fluctuations of the working equipment in the dynamic system of a single-bucket excavator, the rate of passage of the system through the resonance can significantly decrease, which will lead to an increase in dynamic forces; 6) oscillations of the rotation mechanism can have the character of a beating due to fluctuations in the speed of rotation of the turntable and the presence of feedback between the speed and frequency of the disturbing moment; when gearless drive oscillations of the rotation mechanism and working equipment almost do not affect each other.

\section{Conclusions}

The conducted research allows us to draw the following main conclusions:

1. The study of the generalized dynamic system with internal kinematic perturbation in relation to the mechanisms of excavator rotation has shown that the oscillations of the system with the maximum amplitude are generally unstable: after reaching the maximum, the amplitude of the oscillations decreases.

2. The maximum oscillation amplitudes of a generalized dynamical system depend nonlinearly on the amplitude of the internal kinematic perturbation, the damping coefficient, and the ratio of the oscillating masses.

3. When the attenuation coefficient is zero, the oscillations of the generalized dynamic system have the form of beats.

4. In the General case, the fluctuations of the generalized dynamical system are almost periodic. When one of the masses of the system tends to infinity, the oscillations of the system become periodic with a constant amplitude (at a finite attenuation coefficient).

5. With the parameters of the generalized dynamic system corresponding to the mechanisms of rotation of excavators, the estimation of the maximum oscillation amplitudes during the non-stationary process of acceleration of the system can be carried out according to the criteria used for linear systems when passing through the resonance.

A cardinal solution to the problem can be such a design of the mechanism in which the natural frequency of the mechanism lies above the disturbing frequencies or there is no open gear pair at all - the source of the disturbances. The first proposal can be implemented in mechanisms with a gearless drive, and the second-in mechanisms with a central wave gear gearbox, the experience of which is available in crane construction .

The range of options open gear set to reduce its disturbance is associated with the use of a larger number of gear teeth $\mathrm{Z1}$ and applying the reduced ratio of the height of the heads teeth ha* and of such an offset ratio gears, in which the magnitude of the error reduction ratio will depend on the sign of the difference between the steps of gears and wheels.

Improving the performance of the crown pair can also be achieved by improving the methods of heat treatment of gears and the use of new wear-resistant alloys for them.

Improving the accuracy of processing gears of an open gear pair of construction machines can be more expensive than the proposed other methods.

\section{References}

1. R. Sharapov, B.A. Kaytukov, M.A. Stepanov Nekotorye problemy dinamiki i nadezhnosti stroitelnoy tekhniki Mekhanizatsiya stroitelstva V78(7), 5-8 (2017)

2. V.Cherkasov, B.A. Kaytukov, P.D. Kapyrin, V.I. Skel, M.A. Stepanov Reliability of machines and mechanisms. Moscow. NRU MGSU, 272, (2015)

3. Construction machines and equipment of the construction industry: textbook. manual / B. G. Kim [et al.]. Vladimir: VISU Publishing House, 279, (2015) 
4. V. Kuznetsova, V.V. Savinkin Stude of the effect of displacemeent of the contact spot of gear on the durability of the crown of the turntable excavator, Construction and road machines, 1, 12-14, (2017).

5. V. Skel, Open gear train as a source of oscillations of the construction machines mechanisms. IOP Conference Series: Materials Science and Engineering. International Scientific Conference Interstroymeh, ISM 2019. 2020, 012040, (2019)

6. Buckinghem, Earle. Dynamic Loads on Gear Teeth. Published by the American Society of Mechanical Engineers. New York, N.Y. (1931)

7. A. I. Petrusevich, On the calculation of straight-toothed wheels according to Buckingham. "Bulletin of the Metal Industry", 8-9, (1949)

8. D. Kalinin, Y.U. Temis Modelirovanie nelineynykh kolebaniy tsilindricheskikh zubchatykh peredach aviatsionnykh privodov Vestnik Samarskogo gosudarstvennogo aerokosmicheskogo universiteta, V.14(3), 193-202, (2015)

9. L. Grzegorz, I. Michael Friswell, Dynamics of a Gear System with Faults in Meshing Stiffness, Nonlinear Dynamics 41, 415-421, (2005)

10. Dynamic behavior analysis of spur gears with constant \& variable excitations considering sliding friction influence. L. Fuhao, J. Hanjun, L. Shaona, Y. Xuehua, Journal of Mechanical Science and Technology, V. 30, 5363-5370, (2016)

11. S. Ahmed, S.S. Mohamed, R.P. Mohammad, Model-Based Analysis of Spur Gears' Dynamic Behavior in the Presence of Multiple Cracks. Hindawi Journfl Shock and Vibration V. 2018, (2018)

12. O. D. Mohammed, M. Rantatalo, J.-O. Aidanpää, Dynamic modelling of a one-stage spur gear system and vibration-based tooth crack detection analysis, Mechanical Systems and Signal Processing, V. 54, 293-305, (2015)

13. A. Mohamed, S. Sassi, and M. R. Paurobally, Numerical simulation of one-stage gearbox dynamics in the presence of simultaneous tooth crack, in Proceedings of the ICSV24, London, UK, (2017)

14. K. Dimitrion, N. Fokas, Calculation of Spur Gear Dynamic Transmission Error in Consideration of the Progressive Engagement of Compliant Profile-modified Teeth

15. MATEC Web of Conferences 28, 02004, (2015)

16. M. Kurushin, V.B. Balyakin, S.A. Kurushin, Parametric instability of the dynamics of gears. Bulletin of the Samara State Aerospace University. Academician S. P. Korolev (National Research University), No. 5-1 (47), 106-111, (2014)

17. X. Zhao, C. Chen, J. Liu, L. Zhang Dynamic characteristics of a spur gear transmission system for a wind turbine, AMCCE, 1985-1990, (2015)

18. M. Malinkovich, One of the ways to reduce dynamic loads in a gear train, Bulletin of BSTU, No. 2 (22), 78-81, (2009)

19. V. Rudnitskiy, G.D. Moiseev, On the issue of reducing the noise of gears, New materials and technologies in mechanical engineering,, No. 21, 60-63, (2015)

20. L. Kazaz, C. Pfister, P. Ziegler, P. Eberhard, Transient gear contact simulations using a floating frame of reference approach and higher-order ansatz functions. Acta Mech, 231, 1337-1350, (2020)

21. T. Hayasi Nelineynye kolebaniya v fizicheskikh sistemakh, M, Mir, 432, (1968)

22. L. Pars, Analytical dynamics., The main edition of the physical and mathematical literature of the publishing house "Nauka", 636, (1971) 